\title{
Hadron Production in the Nucleus Fragmentation Region
}

\author{
Marcos André Betemps* \\ Conjunto Agrotécnico Visconde da Graça, \\ Universidade Federal de Pelotas \\ Rua Eng. Ildefonso Simoes Lopes, 2791, \\ 96060-290 Pelotas, Rio Grande do Sul, Brazil \\ Maria Beatriz Gay Ducati ${ }^{\dagger}$ \\ Instituto de Física, \\ Universidade Federal do Rio Grande do Sul \\ Caixa Postal 15051, 91501-970 Porto Alegre, \\ Rio Grande do Sul, Brazil \\ (Received on 20 June, 2008)
}

\begin{abstract}
The RHIC hadron production data in hadronic collisions at the forward rapidities may hint the evidence of the Color Glass Condensate (CGC). However, in the opposite region, backward rapidities, new effects should be important in order to describe the observables. In this work, the charged hadron and $\pi^{0}$ productions are investigated in the fragmentation region of the nucleus (backward rapidities) considering $d A u$ and $p p$ collisions in the context of the Color Glass Condensate. In the backward rapidity region, only the proton can be treated as a CGC, and the large $x$ nuclear effects need to be considered in order to describe the cross section. The results are shown by means of the nuclear modification ratio comparing the proton-nucleus and proton-proton cross sections and such ratio presents some dependences on the large $x$ nuclear effects.
\end{abstract}

Keywords: Color Glass Condensate; Backward Rapidities; Hadron Production

\section{INTRODUCTION}

The hadron production in the high energy hadronic collisions at RHIC [1] is one of the recent data that should indicate the existence of the Color Glass Condensate (CGC) [2] in the forward rapidity region. The ratio between deuteriumproton and proton-proton differential cross sections, considering the transverse momentum distributions, is employed to investigate the existence of the Color Glass Condensate. This ratio presents a Cronin enhancement at moderate transverse momentum and more central rapidities, and a suppression at forward ones. The suppression of the ratio with rapidity is related to the quantum evolution of the gluons in the target, and is being considered as one of the evidences of the Color Glass Condensate [3].

Looking to the opposite region in the detector (backward rapidities), new effects like large $x$ nuclear effects or final state effects, should be relevant in order to describe the observables. At this region the nucleus can not be considered as a Color Glass Condensate, once the large $x$ components contribute to the cross section, and only the proton should be treated in this way. Considering the recently measured hadron nuclear modification at backward rapidities, it shows a pronounced peak as more negative values are reached $(\sim-2)$ for $0.5 \mathrm{GeV}<p_{T}<4 \mathrm{GeV}$ [4], which is not well understood. However, as we will show in this work, it should be due to final states interactions.

The main goal of this work is to investigate the hadron production at backward region considering the Color Glass Con-

\footnotetext{
*marcos.betemps@ufpel.edu.br

†beatriz.gay@ufrgs.br
}

densate approach. The CGC theory is well justified at forward rapidities, where the nucleus interacts by means of the small $x$ partons. The use of this approach at the backward kinematical region need to be carefully analyzed. In recent works $[5,6]$ the CGC approach is considered in order to describe the proton-proton collision, which implies to describe the proton as a CGC. If it is so, them we are free to change proton and nucleus in the CGC approach, and investigate the large $x$ nuclear effects in the nucleus, by means of the nuclear parton distributions, while the proton being described by the CGC theory.

Considering this goal, the paper is organized as follows. In the next section, we discuss the hadron production at backward rapidities, presenting all the quantities in order to obtain the cross section for the hadron production. In section 3 we present and discuss the main results of this work and in section 4 the conclusions of the work are presented.

\section{HADRON PRODUCTION}

The hadron production at forward rapidities is investigated in a considerable number of works (for instance $[5,6]$ ). The cross section could be expressed as a convolution of the standard parton distributions for the dilute projectile, the dipolehadron scattering amplitude, in which the high-density effects should be included, and the parton fragmentation functions. The idea in this work is to consider the same cross section at forward rapidities, however, changing the target and the projectile, being now the nucleus interacting at large $x$ the target and the proton at small $x$ the projectile (similar considerations were performed for dilepton production at backward rapidities in the dipole approach [7]). Considering this idea, the cross section for the hadron production at backward rapidities 
in Deuterium-Nucleus collisions, could be written in the form,

$$
\begin{aligned}
\frac{d^{2} \sigma^{d A \rightarrow h X}(\text { Backward })}{d y d^{2} p_{T}} & =\frac{1}{2 \pi} \int_{x_{F}}^{1} d x_{2} \frac{x_{2}}{x_{F}}\left\{f_{q / A}\left(x_{2}, p_{T}\right) N_{F}\left(\frac{x_{2}}{x_{F}} p_{T}, x_{1}\right) D_{h / q}\left(\frac{x_{F}}{x_{2}}, p_{T}\right)\right. \\
& \left.+f_{g / A}\left(x_{2}, p_{T}\right) N_{A}\left(\frac{x_{2}}{x_{F}} p_{T}, x_{1}\right) D_{h / g}\left(\frac{x_{F}}{x_{2}}, p_{T}\right)\right\},
\end{aligned}
$$

where $p_{T}, y$ and $x_{F}$ are the transverse momentum, rapidity and the Feynman- $x$ of the produced hadron, respectively. The variable $x_{1}$ denotes the momentum fraction of a projectile parton, $f\left(x_{1}, p_{T}^{2}\right)$ is the projectile parton distribution functions (nucleus in $d A$ or proton in $p p$ collisions) and $D\left(z, p_{T}^{2}\right)$ represents the parton fragmentation functions into hadrons (we use the KKP fragmentation function [8]). In Eq. (1), $N_{F}(\tilde{\mathbf{k}}, \mathbf{x})$ and $N_{A}(\tilde{\mathbf{k}}, \mathbf{x})$ are the fundamental and adjoint representations of the forward dipole amplitude in momentum space, which represent the probability for scattering of a quark and a gluon of the deuterium (or proton), respectively. Moreover, for this kinematical region $x_{F}=\frac{p_{T}}{\sqrt{s}} e^{-y}$, and the momentum fraction of the target partons is given by $x_{2}=x_{1} e^{-2 y}$.

Concerning the nuclear parton distribution function, we use CTEQ5 [9] as the parton distribution function (PDF) of free protons and the EKS parametrization for the nuclear parton distributions (nPDF) [10, 11]. EKS parameterization gives the nPDF as the free proton PDF multiplied by a factor,

$$
f_{q}^{A}(x, Q)=R_{q}^{A}(x, Q) f_{q}^{p}(x, Q)
$$

and is available only at leading order. We perform the investigation considering other nuclear parton distribution proposed by Sassot and de Florian (nDS) [12] which gives the nPDF as a convolution of the free proton PDF and a weight function:

$$
f_{q}^{A}(x, Q)=\int_{x}^{A} \frac{d y}{y} W_{q}(y, A) f_{q}^{p}\left(\frac{x}{y}, Q\right) .
$$

However, this parametrization is not well behaved at large $x$, as can be seen in the Fig. 1, where the ratio between nuclear and nucleon parton distributions functions $R_{F_{2}}$ is presented. For this reason, the results in this work are obtained with the EKS parametrization. In the Fig. 1 the distinct nuclear effects should be identified: Fermi motion effects $(0.8<x)$, EMC effects $(0.3<x<0.8)$, anti-shadowing $(0.1<x<0.3)$ and shadowing effects $(x<0.1)$.

The dipole scattering amplitude parametrization is considered from the DHJ parametrization [5]. This parametrization was proposed in order to describe the forward region of $d A u$ collisions at RHIC, and present explicit Geometric Scaling violation. The dipole scattering amplitude proposed in the DHJ model was based in the parameterization porposed by Kharzeev, Kovchegov and Tuchin, which incorporates GS violation [13], however, in the DHJ model, this GS violation is

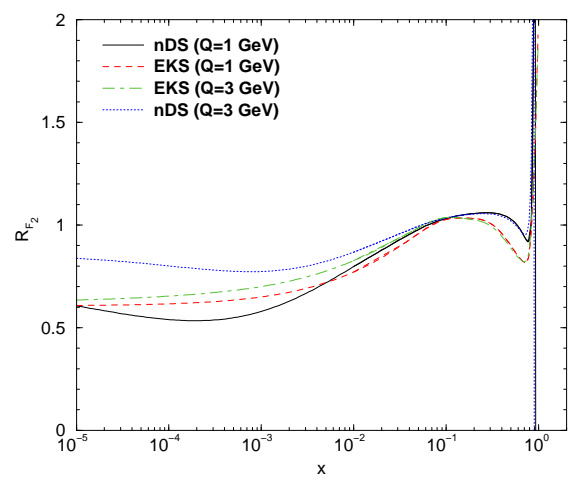

FIG. 1: Comparison between EKS and nDS parametrization at small $Q$. At large $x$ the nDS parametrization predicts negative values for the ratio between nuclear and proton structure functions.

strong. The DHJ model is written in the form,

$$
\begin{aligned}
N_{A}\left(p_{T}, x\right) & =\int d^{2} r e^{i \overrightarrow{p_{T}} \cdot \vec{r}} \\
& \times\left[1-\exp \left(-\frac{1}{4}\left(r^{2} Q_{s}^{2}(x)\right)^{\gamma\left(p_{T}, x\right)}\right)\right] .
\end{aligned}
$$

In this parameterization, the problems that appear in the Fourier transformation [14] are prevented by the $p_{T}$ dependence of the $\gamma$ instead of the $r$ dependence. The expression for $N_{F}$ is obtained by means of the replacement $r^{2} Q_{s}^{2}(x) \rightarrow$ $\left(C_{F} / C_{A}\right) r^{2} Q_{s}^{2}$, where $C_{F} / C_{A}=4 / 9$. The anomalous dimension $\gamma$ of the DHJ parameterization has the form,

$$
\begin{aligned}
& \gamma\left(p_{T}, x\right)=\gamma_{s}+\Delta \gamma\left(p_{T}, x\right) \\
& \gamma\left(p_{T}, x\right)=\gamma_{s}+\left(1-\gamma_{s}\right) \frac{\log \left(p_{T}^{2} / Q_{s}^{2}(x)\right.}{\lambda y+d \sqrt{y}+\log \left(p_{T}^{2} / Q_{s}^{2}(x)\right)},
\end{aligned}
$$

where $y=\log 1 / x$. The saturation scale $Q_{s}$ and the parameter $\lambda$ are taken from the GBW model [15]. In this parameterization, $d=1.2$ is determined by the fit procedure to the RHIC data on hadron production and $\gamma_{s}=0.628$. The GS violation in the DHJ model is represented by the term $\Delta \gamma\left(p_{T}, x\right)$ in the equation 5 .

Having presented all the components in order to obtain the cross section for the hadron production at backward rapidities, in what follows the cross section could be evaluated considering $p p$ and $d A u$ collision at RHIC energies, analyzing the charged hadron and $\pi^{0}$ productions. In the Fig. 2 the spectra 
for $\pi^{0}$ production is presented as a function of the transverse momentum of the produced hadron.

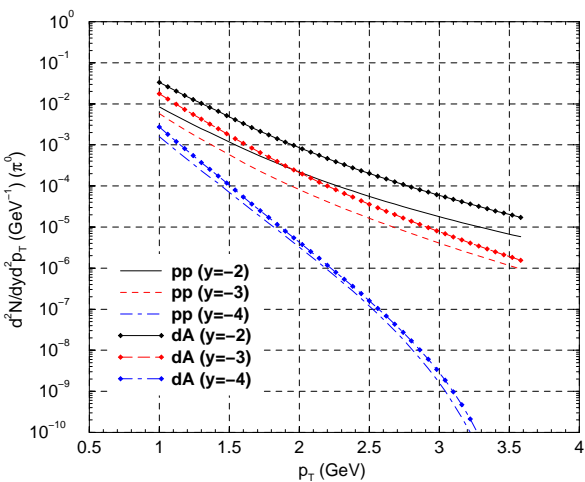

FIG. 2: $\pi^{0}$ production at RHIC energies in $p p$ and $d A u$ collisions.

Analyzing the Fig. 2 we verify the expected behavior, the spectra is enlarged to more central rapidities (the behavior is similiar for charged hadron production). In these calculations no $K$ factor is under consideration (the $K$ factor should simulate NLO contributions). It should be interesting to address that the calculations performed here are justified only at backward rapidities. It should not describe the cross section in the central rapidity region, since at this kinematical regime, the proton should not be described as a Color Glass Condensate. It is an approximation, which is considered reasonable only at backward or forward rapidities.

Having discussed the hadron production at the backward rapidities, in the next section the nuclear modification ratio is investigated at this kinematical region.

\section{RESULTS AND DISCUSSIONS}

The cross section presented in this work is evaluated in the LO calculation. In order to describe the data, a $K$ factor should be employed. It should be verified that in the forward rapidities the $p p$ and $d A u$ spectra are described considering the same $K$ factor at same rapidity and energy. This implies that the production mechanism is the same considering $p p$ and $d A u$ processes at forward rapidities, and the saturation effects are being considered in both cases. In the backward rapidities, new effects are present in the nucleus (large $x$ effects), which are not present in the proton. This should be the main approximation realized in this work: we consider the same $K$ factor in $p p$ and $d A u$ collision at backward rapidities, in order to investigate the nuclear modification factor, which is defined in the form,

$$
R_{d A}=\frac{d^{2} N(d A) / d y d^{2} p_{T}}{d^{2} N(p p) / d y d^{2} p_{T}}
$$

In this definition, we have not included any normalization factor. The normalization of the ratio $R_{d A}$ is a crucial point of this investigation, however, we intend to analyse this aspect in a complete version of this work, which is in progress [16]. At the moment, we will investigate only the $p_{T}$ dependence of the ratio $R_{d A}$ considering $\pi^{0}$ and charged hadron productions. In the Fig. 3 and 4 the nuclear modification ratio is investigated for these observables at RHIC energies $\sqrt{s}=200 \mathrm{GeV}$ and backward rapidities $y=-2,-3,-4$. We consider the CTEQ5 LO parametrization for the free proton, deuterium without nuclear effects and EKS parametrization for the nuclear parton distributions.

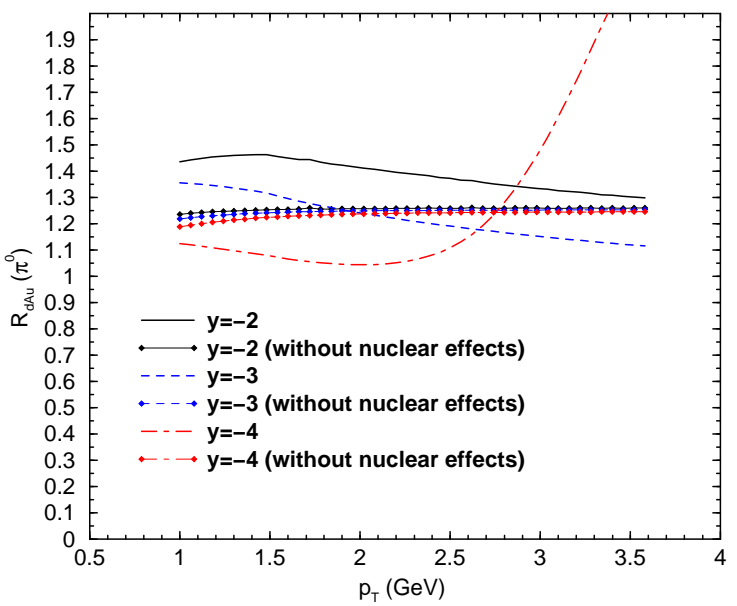

FIG. 3: Nuclear modification ratio for the $\pi^{0}$ production at RHIC energies.

In order to identify the large $x$ effects in the nuclear modification factor, we perform calculations with and without nuclear effects. In the cross section (Eq. 1), the integration over $x_{2}$ with the limits $x_{F}$ and 1, implies that all the nuclear effects for $x>x_{F}$ are contributing to the cross section. Only concerning the fragmentation function the results presented here are distinct considering charged hadron and $\pi^{0}$ productions.

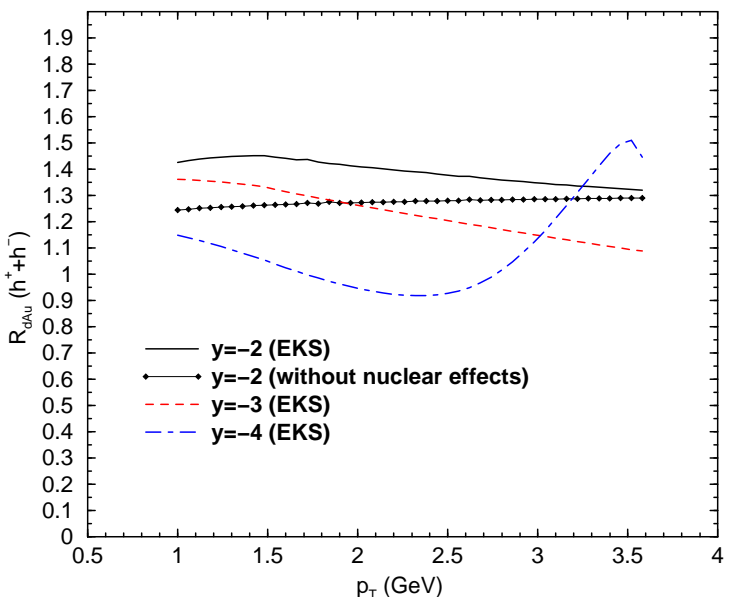

FIG. 4: Nuclear modification ratio for the charged hadron production at RHIC energies.

In the Fig. 3 the nuclear modification factor for the $\pi^{0}$ production is presented. For the rapidity $y=-2$ the nuclear effects provide an increment on the nuclear modification ratio. 
This behavior is due to the large $x$ effects present in this region. The anti-shadowing, EMC and Fermi motion are contributing to the cross section and the net result is an increment of the nuclear cross section, since the $R_{F_{2}}$ is larger than 1 for anti-shadowing and Fermi motion regions. For $y=-3$ the nuclear effects provide a reduction of the nuclear modification ration at large $p_{T}$ (EMC effect) and an increment on the $R_{d A}$ at small $p_{T}$ (anti-shadowing effect). Considering very backward rapidities, the nuclear effects implies that the $R_{d A}$ is reduced at small $p_{T}$ (EMC effect) and present a peak at large $p_{T}$, which is due to the dominance of the Fermi motion effect.

In the Fig. 4 the nuclear modification factor for the charged hadron production is presented. The conclusions considering the charged hadron are the same obtained in the $\pi^{0}$ production, once the calculation are distinct only concerning the fragmentation function.

\section{CONCLUSIONS}

In this work, the large $x$ nuclear effects (Fermi motion, EMC and antishadowing) provide a reduction of the nuclear modification ratio in some RHIC kinematical regions, however, these effects should implie in an increment in the ratio $R_{d A}$ for other regions. The behavior of the ratio $R_{d A}$ is similar considering $\pi^{0}$ and charged hadron productions. We verify that the nuclear effects at backward rapidities at RHIC energies provide an increment on the ratio $R_{d A}$ for $y=-2$. For $y=-3$, the nuclear effects produce an increment in the ratio $R_{d A}$ at small $p_{T}$ and a reduction of the ratio at large $p_{T}$. Considering $y=-4$, only at large $p_{T}$ the large $x$ nuclear effects provides a strong increment on the nuclear modification factor, which is associated to the Fermi motion nuclear effect.

The results obtained in this work should be interesting to compare with the nuclear modification factor data at RHIC backward rapidities for the hadron production [4] (the data present the nuclear modification factor from the central rapidites up to $y=-2$ ). This investigation will be present in a complete version of this work, which includes a comparison with the RHIC data and an estimation of the magnitude of the final state effects contributions. The results presented in this contribution should be contrasted with eletromagnetic observables at this kinematical region [7], which should be another way of investigation of the large $x$ nuclear effects, that we intend to address in a near future.

\section{Acknowledgments:}

We would like to thanks to all participants of the II Latin American Workshop on High Energy Phenomenology due to the excellent discussions. This work was partially financed by the Brazilian funding agencies CNPq and FAPERGS.
[1] I. Arsene et al. [BRAHMS Collaboration], Phys. Rev. Lett. 91, 072305 (2003); Phys. Rev. Lett. 93, 242303 (2004); Phys. Rev. Lett. 94, 032301 (2005);

J. Adams et al. [STAR Collaboration], Phys. Rev. Lett. 97, 152302 (2006).

[2] E. Iancu, A. Leonidov and L. McLerran, Nucl.Phys. A692, 583 (2001); E. Ferreiro, E. Iancu, A. Leonidov and L. McLerran, Nucl. Phys. A701, 489 (2002); J. Jalilian-Marian, A. Kovner, L. McLerran and H. Weigert, Phys. Rev. D 55, 5414 (1997); J. Jalilian-Marian, A. Kovner and H. Weigert, Phys. Rev. D 59, 014014 (1999); ibid. 59, 014015 (1999); ibid. 59, 034007 (1999).

[3] J. L. Albacete, N. Armesto, A. Kovner, C. A. Salgado and U. A. Wiedemann, Phys. Rev. Lett. 92, 082001 (2004).

[4] S.S. Adler, et al. PHENIX Collaboration. Phys. Rev. Lett. 94, $082302(2005)$

[5] A. Dumitru, A. Hayashigaki and J. Jalilian-Marian, Nucl. Phys. A 765, 464 (2006); Nucl. Phys. A 770, 57 (2006).

[6] D. Boer, A. Utermann, E. Wessels, arXiv:0711.4312 [hep-ph].
[7] M. A. Betemps, M. B. Gay Ducati and E. G. de Oliveira, Phys. Rev. D 74, 094010 (2006)

[8] B. A. Kniehl, G. Kramer and B. Potter, Nucl. Phys. B 582, 514 (2000).

[9] H. L. Lai et al. [CTEQ Collaboration], Eur. Phys. J. C 12, 375 (2000).

[10] K. J. Eskola, V. J. Kolhinen and C. A. Salgado, Eur. Phys. J. C 9, 61 (1999).

[11] K. J. Eskola, V. J. Kolhinen and P. V. Ruuskanen, Nucl. Phys. B 535, 351 (1998).

[12] D. de Florian and R. Sassot, Phys. Rev. D 69, 074028 (2004).

[13] D. Kharzeev, Y. V. Kovchegov, K. TUchin, Phys. Lett. B 599, 23 (2004).

[14] M. A. Betemps and M. B. Gay Ducati, Phys. Rev. D 70 (2004) 116005; Phys. Lett. B 636, 46 (2006).

[15] K. Golec-Biernat, M. Wusthoff, Phys. Rev. D 59, 014017 (1999); ibid. 60, 114023 (1999).

[16] M. A. Betemps, M. B. Gay Ducati, work in progress. 